\title{
VIEWPOINT
}

\section{Towards a deeper insight into strongly correlated electron systems - the symbiosis between experiment and theory}

\author{
Peter Fischer \\ Center for X-ray Optics \\ Lawrence Berkeley National Laboratory \\ Berkeley, CA 94720 U.S.A. \\ PJFischer@lbl.gov
}

It is generally accepted that many of the fundamental properties of the solid state - in particular electronic, magnetic and thermal properties - ultimately depend on electronic correlations. Strongly correlated electron systems give rise to metal-insulator transitions, halfmetallicity, heavy fermion systems etc., all of which are lively topics of research in modern solid state physics.

Scientists are fascinated by the variety of features that solid state materials exhibit, such as ferromagnetism or superconductivity which challenge a fundamental explanation, but have also a huge technological impact. Ferromagnetic materials can be seen as a prototype of applied nanoscience which everybody experiences in the form of common ultrahigh density magnetic storage media. On the other hand, the effect of superconductivity with the promise to contribute to a lossless energy transport, if it could operate at high enough temperatures, is still in a state of infancy with respect to potential applications. A similar situation can be seen in the quest to design novel smart materials, such as multiferroics, which would allow to control e.g. magnetization by electric potentials [1].

One of the fundamental questions in solid state physics relates to the possible coexistence of ferromagnetism and superconductivity. Although both being an effect of long-range ordering, they seem to be mutually exclusive, which is heavily debated. It seems to be obvious, that the ferromagnetic moment gives rise to an internal magnetic field strong enough to break the Cooper pairing, which is required to form the superconducting phase. However, magnetic superconductors have been discovered, and are now being investigated experimentally and theoretically [2].

The analytical tools, which are required to investigate experimentally with high accuracy and sensitivity effects in strongly correlated systems, have seen major achievements over the last decade, and X-rays as an ideal probe have taken over a dominant role. The rapidly increasing number of synchrotron radiation laboratories worldwide provide high brilliant $\mathrm{X}$-ray sources with tunable wavelength regimes, outstanding polarization characteristics and with an increase in photon intensity that has surpassed even Moore's law in its growth. Sophisticated experimental X-ray techniques that require high flux, brightness, coherence and polarization have thus become feasible and are commonly in use nowadays.

Angle Resolved PhotoEmission Spectroscopy (ARPES) is such an example. Mapping electronic bandstructures in High- $\mathrm{T}_{\mathrm{c}}$ superconductors with this state-of-the-art technique allows now to see features in detail within a reasonable amount of time $[3,4,5]$.

Similarly, X-ray absorption spectroscopy which probes the local electronic structure is common at all synchrotron facilities worldwide. Measuring the X-ray absorption in a ferromagnetic solid with circularly polarized X-rays has become an inevitable powerful experimental technique for studies of nanomagnetism. Physically, it is based on the effect of $\mathrm{X}$-ray magnetic circular dichroism (XMCD), which describes the fact, that the X-ray absorption cross section depends strongly and in an element specific manner on the scalar 
product between the helicity of the photons and the magnetization of the specimen. Even more, the application of magneto-optical sum rules to experimentally observable X-ray absorption spectra gives - in a unique way - access to fundamental magnetic ground state properties, such as local spin and orbital moment [6]. Hence convincing experimental evidence of magnetic properties of a solid is provided by XMCD based X-ray spectroscopies [7] and X-ray microscopies [8,9]

But experimental findings of even highest accuracy require a solid theoretical description. Concomitantly to the experimental achievements, the theoretical description of the solid state has seen significant improvements of equal level.

A successful first-principle method to study theoretically ground-state properties of many electron system is the density functional theory within the local spin density approximation. To account for systems with strong Coulomb correlations occurring in strongly correlated systems, extensions were developed such as the LDA+U method [10], and are now capable to describe such systems.

A recent paper by V.N.Antonov et al [11] is an excellent demonstration of the symbiosis of theory with experiment and the achievements that can be obtained. They investigated $\mathrm{UGe}_{2}$, one of the systems which has been discovered recently to exhibit both superconductivity and ferromagnetism at high pressure. Based on high quality experimental XMCD data they could convincingly show that excellent agreement between the theoretical calculation and experimentally measured XMCD spectra could be obtained with the LSDA+U approximation. Furthermore their calculations were also consistent with the magnetic moments in $\mathrm{UGe}_{2}$. Even the K edge results, which are generally of minor interest both theoretically and experimentally due to the much weaker spin-orbit coupling in the initial state yielding to minute XMCD signals, could be reproduced very well.

Do we really understand strongly correlated electron systems? The quest is still open and novel and smart materials exhibiting more fascinating and technologically relevant properties are still waiting to be discovered.

\section{References}

[1] N. Spalding and M. Fiebig 2005 Science 309391

[2] see e.g. Z F Banfield, J A Duffy, J W Taylor, C A Steer, A Bebb, M J Cooper, L Blaauw, C Shenton-Taylor and R Ruiz-Bustos J. Phys.: Condens. Matter 200517 5533;

T. Park, F. Ronning, H. Q. Yuan, M. B. Salamon, R. Movshovich, J. L. Sarrao and J. D. Thompson, Nature 2006440 65-68;

T.-K. Ng and W.-T. Leung 2001 Europhys. Lett. 53653

[3] G.-H. Gweon, T. Sasagawa, S.Y. Zhou, J. Graf, H. Takagi, D.-H. Lee and A. Lanzara, 2004 Nature 430187

[4] N. Mannella, W. L. Yang, X. J. Zhou, H. Zheng, J. F. Mitchell, J. Zaanen, T. P. Devereaux, N. Nagaosa, Z. Hussain and Z.-X. Shen 2005 Nature 438474

[5] K. Byczuk, M. Kollar, K. Held, Y.-F. Yang, I. A. Nekrasov, Th Pruschke and D. Vollhardt, 2007 Nature Physics 3168

[6] B. T. Thole, P. Carra, F. Sette, and G. van der Laan, 1992 Phys. Rev. Lett. 68, 1943; P. Carra, B. T. Thole, M. Altarelli, and X. Wang 1993 Phys. Rev. Lett. 70, 694

[7] C. T. Chen, F. Sette, Y. Ma, and S. Modesti, 1990, Phys. Rev B 427262

[8] Stoehr, J. Wu Y, Hermsmeier B D, Samant M G, Harp G R, Koranda S, Dunham D, Tonner B P 1993 Science 259658

[9] P. Fischer, G. Schütz, G. Schmahl, P. Guttmann und D. Raasch, 1996 Z.f. Physik B 101 313 
[10] V.I. Anisimov, F Aryasetiawanz and A I Lichtenstein, 1997 J. Phys.: Condens. Matter 9 767

[11] V.N. Antonov, B N Harmon and A N Yaresko 2007 J. Phys.: Condens. Matter in press 